\title{
An active alternative splicing isoform of human mitochondrial 8-oxoguanine DNA glycosylase (OGG1)
}

Chie Furihata ${ }^{1,2}$

\begin{abstract}
Eight alternatively spliced isoforms of human 8-oxoguanine DNA glycosylase (OGG1) (OGG1-1a, -1b, -1c, -2a, -2b, $-2 c,-2 d$ and $-2 e)$ are registered at the National Center for Biotechnology Information (NCBI). OGG1-1a is present in the nucleus, whereas the other seven isoforms are present in the mitochondria. Recombinant OGG1-1a has been purified and enzyme kinetics determined. OGG1(s) in mitochondria have not been fully characterized biochemically until recently. The major mitochondrial OGG1 isoform, OGG1-2a (also named $\beta-O G G 1$ ), has also been expressed and purified; however, its activity is unresolved. Recently, we purified recombinant mitochondrial OGG1-1b and found that it was an active OGG1 enzyme. We reported its enzyme kinetics and compared the results with those of OGG1-1a. The reaction rate constant of OGG1-1b 8-oxoG glycosylase activity $\left(k_{\mathrm{g}}\right)$ was 8-oxoG:C > > 8-oxoG:T > > 8-oxoG:G > 8-oxoG:A and was similar to that of OGG1-1a under single-turnover conditions ([E] > [S]). Both OGG1-1b and OGG1-1a showed high specificity towards 8-oxoG:C. The reaction rate constant of OGG1-1b N-glycosylase/DNA lyase activity $\left(k_{g l}\right)$ was 8-oxoG:C > 8-oxoG:T $\simeq$ 8-oxoG:G > > 8-oxoG:A and that of OGG1-1a was 8-oxoG:C > 8-oxoG:T, 8-oxoG:G and 8-oxoG:A. The $k_{\mathrm{gl}}$ of OGG1-1b and OGG1-1a is one order of magnitude lower than the corresponding $\mathrm{kg}_{\mathrm{g}}$ value. OGG1-1b showed an especially low $k_{\mathrm{gl}}$ towards 8-oxoG:A. Comparable expression of OGG1-1a and OGG1-1b was detected by RT-PCR in normal human lung tissue and lung cell lines. These results suggest that OGG1-1b is associated with 8-oxoG cleavage in human lung mitochondria and that the mechanism of this repair is similar to that of nuclear OGG1-1a. Currently, the other five mitochondrial OGG1 isoforms have not been isolated. I summarize information on OGG1 isoform mRNAs, coding DNA sequences and amino acid sequences that are archived by the National Center for Biotechnology Information.
\end{abstract}

Keywords: Human 8-oxoguanine DNA glycosylase, OGG1, Mitochondrial OGG1, OGG1-1a, OGG1-1b, OGG1-2a

\section{Introduction}

According to the National Center for Biotechnology Information (NCBI), the human 8-oxoguanine DNA glycosylase (OGG1) gene encodes the enzyme responsible for the excision of 8-oxoguanine (8-oxoG), a mutagenic base byproduct that occurs as a result of exposure to reactive oxygen (http://www.ncbi.nlm.nih.gov/gene/4968). 8-oxoG was first described in 1984 by Kasai et al. [1] and is an abundant DNA adduct caused by oxidative stress [2]. The action of OGG1 includes lyase activity for chain cleavage.

Correspondence: chiefurihata@gmail.com

'School of Science and Engineering, Aoyama Gakuin University, Sagamihara, Kanagawa 252-5258, Japan

2Division of Molecular Target and Gene Therapy Products, National Institute of Health Sciences, Setagayaku, Tokyo 158-8501, Japan
In 1997 Aburatani et al. described four isoforms (OGG1$1 \mathrm{a},-1 \mathrm{~b},-1 \mathrm{c}$ and -2) [3], and then in 1999, Nishioka et al. described seven isoforms $(-1 \mathrm{a},-1 \mathrm{~b},-2 \mathrm{a},-2 \mathrm{~b},-2 \mathrm{c},-2 \mathrm{~d}$ and -2e) [4]. They classified these isoforms into two groups based on their last exon: type 1 isoforms end with exon 7 and type 2 isoforms end with exon 8 . At present, their type 1 nomenclature cannot be applied to OGG1-1b because it contains only exons 1-6 (NCBI: NM_016819). Now, type 1 and type 2 OGG1s can be grouped with or without exon 8 . Eight alternatively spliced isoforms of OGG1 (OGG1-1a, -1b, -1c, -2a, -2b, -2c, -2d and $-2 \mathrm{e}$ ) are registered in the NCBI gene and nucleotide database. OGG1-1a is the only OGG1 present in the nucleus [4], whereas the other seven isoforms have been shown to be present in mitochondria [3-5]. Recombinant 
OGG1-1a has been purified and its enzyme kinetics determined [6-8]. Although mitochondrial OGG1 was suggested to have a crucial role against mitochondrial DNA damage [9], the responsible OGG1 splicing isoform(s) have not been described in detail. Recombinant production of the mitochondrial major OGG1 isoform, OGG1-2a (also named $\beta$-OGG1), has been carried out; however, the activity of this OGG1 was very low [9] or un-detectable [10]. Recently, we purified recombinant mitochondrial OGG1-1b and showed that it was an active OGG1 enzyme; we determined its enzyme kinetics, and compared these results with those of OGG1-1a [11]. Similar 8-oxoG glycosylase activity and $\mathrm{N}$-glycosylase/DNA lyase activity was detected except for $k_{\mathrm{gl}}(N$-glycosylase/DNA lyase activity) against 8-oxoG:A. Currently, the other five mitochondrial OGG1 isoforms have not been purified.

A review of all eight alternatively spliced isoforms has not been published; therefore, in this review I present the published data on mainly mitochondrial OGG1 isoforms and summarize the information on eight alternative splicing isoforms archived by the NCBI.

\section{OGG1-1b}

Human OGG1-1b was cloned as an alternatively spliced isoform of OGG1 by Abratani et al. in 1997 [3] and confirmed by Nishioka et al. [4]. They proposed that the OGG1-1b mRNA contained an extra 244 bp from intron 6 and the same exon 7 compared with the OGG1-1a mRNA. However, OGG1-1b mRNA is currently described in NCBI (NM_016819) as composed of 6 exons (exons 1-6) and does not possess exons 7 and 8. Localization of the OGG1$1 \mathrm{~b}$ protein in mitochondria was published by Takao et al. [5]. They showed localization of a FLAG-tagged OGG1-1b in the mitochondria of COS-7 cells by immunofluorescence staining. The expression of OGG1-1b was demonstrated by RT-PCR in several human tissues, including lung [11, 12], colon [3], cerebrum [4], kidney [4], fetal brain [4], peripheral blood lymphocytes [13], and in human cell lines including normal-derived lung cell MRC-9, lung cancer cell lines, H23, H69, Lu65, Lu135, PC10 and PC13 [12], A549, ABC-1, EBC-1, LK-2, LU99 and RERF-LC-MA [11], Jurkat cells (a human T-cell leukemia cell line) [4], and an immortalized line of T-lymphocyte cells [14].

Recently, we purified recombinant OGG1-1b and OGG1-1a using commercial human lung total RNA as the starting material, and showed that OGG1-1b was an active OGG1 enzyme. We compared the enzyme kinetics of mitochondrial OGG1-1b with the nuclear OGG1-1a protein [11], as described in the next section.

\section{Comparison of enzyme kinetics between OGG1-1b and OGG1-1a}

The reaction rate constants for 8-oxoG glycosylase activity $\left(k_{\mathrm{g}}\right)$ and $\mathrm{N}$-glycosylase/AP activity $\left(k_{\mathrm{gl}}\right)$ were determined under single-turnover conditions $([\mathrm{E}]>[\mathrm{S}])$ of OGG1-1a and OGG1-1b with $100 \mathrm{nM}$ enzyme and $20 \mathrm{nM}$ substrate [11]. Alexa 555-labeled 36-mer oligonucleotide substrates (8OG_36_Alexa: [Alexa 555]GGAATTCCTCGAGGT[8oxoG]GACGGTATCCGATGGCCGCT, c-16C: AGCGGC CATCGGATACCGTCCACCTCGAGGAATTCC, c-16A: AGCGGCCATCGGATACCGTCAACCTCGAGGAATT CC, c-16G: AGCGGCCATCGGATACCGTCGACCTC GAGGAATTCC and c-16 T: AGCGGCCATCGGATAC CGTCTACCTCGAGGAATTCC) were used. The $k_{\mathrm{g}}$ of the 8-oxoG glycosylase activity of both OGG1-1b and OGG1-1a was 8-oxoG:C > > 8-oxoG:T > > 8-oxoG:G > 8-oxoG:A (7.96, 0.805, 0.070, and $0.015 \mathrm{~min}^{-1}$, respectively for OGG1-1b, and 7.21, 1.37, 0.125, and $0.031 \mathrm{~min}^{-1}$, respectively for OGG1-1a). The enzymes show similar kinetic values. Both OGG1-1b and OGG1-1a showed high specificity towards 8-oxoG:C. The $k_{\mathrm{gl}}$ of OGG1-1b $\mathrm{N}$-glycosylase/DNA lyase activity was 8 -oxoG:C $>8$ oxoG:T $\simeq 8$-oxoG:G > > 8-oxoG:A $(0.286,0.079,0.040$, and $\sim 0.00 \mathrm{~min}^{-1}$, respectively) and that of OGG1-1a was 8-oxoG:C > 8-oxoG:T, 8-oxoG:G and 8-oxoG:A $\left(0.254,0.083,0.075\right.$, and $0.072 \mathrm{~min}^{-1}$, respectively). The reaction rate constant of $k_{\mathrm{gl}}$ of OGG1-1b and OGG1-1a was one order of magnitude lower than that of their $k_{\mathrm{g}}$ values. OGG1-1b showed a particularly small $k_{\mathrm{gl}}$ towards 8-oxoG:A, and an exact numerical value of $k_{\mathrm{gl}}$ for OGG1-1b could not be calculated from the experimental conditions employed [11]. Similar multipleturnover kinetics data $\left(A_{0}, k_{\mathrm{obs}}\right.$ and $\left.k_{\mathrm{ss}}\right)$ under $[S]>[E]$ for OGG1-1b and OGG1-1a against the 8-oxoG:C substrate were observed. Similar substrate specificity of OGG1-1b and OGG1-1a against 8-oxoG:C and 8oxoG:A was observed. Product formation was higher against 8-oxoG:C than 8-oxoG:A for OGG1-1b and OGG1-1a. APEX nuclease 1 (APEX1; NM_001641) was required to promote DNA strand breakage by OGG1-1b. These results suggest that OGG1-1b is associated with 8-oxoG cleavage in human lung mitochondria and that the mechanism of this repair is similar to that of nuclear OGG1-1a.

\section{Active site amino acids}

Various amino acids in the active site of OGG1-1a have been proposed, including, Gly-42, Asn-149, An-150, Lys249, Cys-253, Asp-268, Gln-315, Phe-319 [6], His-270, Gln-315, Asp-322 [15], Arg-154, Val-317, Phe-319 [10], Arg-46, Arg-131, and Arg-154 [16]. Hashiguchi et al. compared glycosylase activity of OGG1-1a and OGG1-2a by site-directed mutagenesis and suggested that Val-317 is a critical residue for glycosylase activity [10]. OGG1-1b protein is identical to OGG1-1a protein from amino acid 1 to 317, including Val-317, and is an active OGG1 [11] despite not possessing Phe-319 and Asp-322. OGG1-2a protein is identical to OGG1-1a protein from amino acid 
1 to 316 but does not possess Val-317, Phe-319, or Asp322 and its enzyme activity is low [9] or not detectable [10]. These results suggest that Val-317 is a critical residue for glycosylase activity. Other OGG1 isoforms have not been purified and their enzyme activities have not been determined.

\section{OGG1-2a}

Human OGG1-2 (now OGG1-2a) was cloned as an alternatively spliced isoform of OGG1 in 1997 by Abratani et al. [3] and Roldán-Arjona et al. [9]. Localization of OGG1-2a protein in mitochondria was demonstrated in COS-7 cells [4] and HeLa MR cells [3]. In addition, the expression of OGG1-2a was demonstrated by northern blot analysis and by RT-PCR in various tissues [3].

Inconsistent findings regarding OGG1-2a protein have been published. Hashiguchi et al. [10] purified recombinant OGG1-2a ( $\beta$-OGG1) and reported that OGG1-2a did not show any significant OGG1 activity in vitro. They examined OGG1 activity with $100 \mathrm{nM}$ OGG1-2a and $10 \mathrm{nM}$ oligonucleotide as the substrate, and found no activity. In the control experiment, they examined 1 nM OGG1-1a and 10 nM oligonucleotide substrate and found active OGG1 activity. Roldán-Arjona et al. [9] reported the purification of recombinant OGG1-2a and showed OGG1 activity against 8-oxoG:C oligonucleotide with $1 \mu \mathrm{M}$ enzyme and $5 \mathrm{nM}$ substrate. The OGG1 activity of OGG1-2a in this experiment was very low, because they used an unusually high enzyme concentration.

Recently, Su et al. suggested that OGG1-2a (written as $\beta$ OGG1) was an accessory factor in mitochondrial Complex I function and was related to mitochondrial base excision repair [17].

\section{Other mitochondrial isoforms}

OGG1-1c was cloned as an alternative splicing isoform of OGG1 in 1997 by Abratani et al. [3]. Expression of OGG1-1c was demonstrated by RT-PCR in some human tissues including the colon [3]. Localization was demonstrated by expressing epitope-tagged OGG1-1c in COS-7 cells [5]. The expression of OGG1-2b, $-2 \mathrm{c},-2 \mathrm{~d}$, and $-2 \mathrm{e}$ was demonstrated by RT-PCR in a small number of human tissues including the cerebrum and kidney, and in the Jurkat cell line by Nishioka et al. [4]. These proteins have not been purified.

\section{Analysis of information on the eight alternatively spliced isoforms of $O G G 1$ archived with the NCBI}

Table 1 summarizes the mRNA accession number, nucleotide (nt) length, position of the $5^{\prime}$-UTR, coding DNA sequence (CDS) and 3'-UTR, exons, position of exon 1, 2, 3, 4, 5, 6, 7, and 8 of the eight alternative splicing isoforms of OGG1, as derived from the gene and nucleotide database of the NCBI (http://www.ncbi.nlm.nih.gov/gene/
4968). Table 2 summarizes alternative splicing isoforms of OGG1 CDS, nt length, identity to OGG1-1a CDS and identity to OGG1-2a CDS according to the NCBI and examined by BLAST (http://blast.ncbi.nlm.nih.gov/Blast.cgi). Table 3 summarizes the protein accession number, amino acid length, identity to the OGG1-1a protein, identity to the OGG1-2a protein (as determined by BLAST), position of mitochondrial and nuclear localization signals, and OGG1 activity.

The OGG1-1a mRNA has exons 1-7 and no exon 8. The CDS begins at nt 344 in exon 1 , and the nt sequence of 1292-1381 (90 bp) in exon7 is the last part of the CDS. The OGG1-1a CDS is composed of part of exon 1, all of exons 2, 3, 4, 5, 6 and part of exon 7 .

Exon 6 is the last exon of OGG1-1b mRNA. The nt sequence 1242-1318 (77 bp) in exon 6 is the last part of the CDS. Although the nt sequence of 1536-1882 (347 bp) in exon 6 of the OGG1-1b mRNA is the same as the entire exon 7 of the OGG1-1a mRNA (nt sequence 1292-1638, $347 \mathrm{bp}$ ), the former represents part of the $3^{\prime}$-UTR. As for the OGG1-1b CDS, the nt sequence of 1242-1291 from exon 6 of the OGG1-1b mRNA is identical to the whole exon 6 CDS of the OGG1-1a mRNA. The OGG1-1b mRNA nt sequence of $1292-1294$ is identical to the first part of the exon 7 CDS of the OGG1-1a mRNA. The OGG1-1b mRNA 1295-1318 sequence (24 bp), which encodes seven amino acids and the stop codon, differs from the 1295-1318 sequence (CDS) from exon 7 of the OGG1-1a mRNA, resulting in a different amino acid sequence for the last seven amino acids of OGG1-1b when compared with the OGG1-1a sequence.

The OGG1-1c mRNA has exon 7, but the nt sequence of this exon is different from that of OGG1-1a mRNA. It also has no exon 8 . The nt sequence 1292-1576 (285 bp) from exon 7 of the OGG1-1c mRNA is the last part of the CDS, but differs from the 1292-1381 CDS (90 bp) from exon 7 of the OGG1-1a mRNA. The nt sequence of 1309-1398 (90 bp) of the OGG1-1c mRNA, a part of the CDS from exon 7, is identical to 1292-1381 (90 bp) of the OGG1-1a mRNA, the entire exon $7 \mathrm{nt}$ sequence.

Only type 2 OGG1 mRNAs have exon 8 . All type 2 OGG1 mRNAs have the same exon 8 nt sequence (861 bp). The OGG1-2a mRNA has no exon 7. The nt sequence 1292-1618 (327 bp) in exon 8 of the OGG1-2a mRNA is the last part of the CDS and the sequence 1619-2158 (540 bp) is the 3' UTR.

The OGG1-2b mRNA has no exons 5-7. The nt sequence 1091-1417 (327 bp) in exon 8 of the OGG1-2b mRNA is the last part of the CDS and is identical to the OGG1-2a CDS, resulting in an identical amino acid sequence for the last 108 amino acids of OGG1-2a and OGG1-2b.

The OGG1-2c mRNA has no exons 4-7. The nucleotide sequence 909-931 (23 bp: the first two nucleotides cross a 
Table 1 Alternative splicing isoforms of OGG1mRNA according to NCBI

\begin{tabular}{|c|c|c|c|c|c|c|c|c|c|c|c|c|c|c|c|}
\hline Type & Name & $\begin{array}{l}\text { mRNA } \\
\text { accession }\end{array}$ & nt bp & 5'-UTR & CDS & 3'-UTR & Exons & Exon 1 & Exon 2 & Exon 3 & Exon 4 & Exon 5 & Exon 6 & Exon 7 & Exon 8 \\
\hline 1 & OGG1-1a & NM_002542 & 1652 & $1-343$ & 344-1381 & $1382-1652$ & $1,2,3,4,5,6,7$ & $1-480$ & $481-728$ & 729-908 & $909-1090$ & $1091-1241$ & $1242-1291$ & $1292-1638$ & No \\
\hline 1 & OGG1-1b & NM_016819 & 1896 & $1-343$ & 344-1318 & 1319-1896 & $1,2,3,4,5,6$ & $1-480$ & $481-728$ & 729-908 & 909-1090 & $1091-1241$ & $1242-1882$ & No & No \\
\hline 1 & OGG1-1C & NM_016820 & 1669 & $1-343$ & $344-1576$ & 1577-1669 & $1,2,3,4,5,6,7$ & $1-480$ & $481-728$ & 729-908 & 909-1090 & $1091-1241$ & $1242-1291$ & 1292-1655 & No \\
\hline 2 & OGG1-2a & NM_016821 & 2158 & $1-343$ & $344-1618$ & $1619-2158$ & $1,2,3,4,5,6,8$ & $1-480$ & $481-728$ & 729-908 & $909-1090$ & $1091-1241$ & $1242-1291$ & No & $1292-2152$ \\
\hline 2 & OGG1-2b & NM_016826 & 1957 & $1-343$ & $344-1417$ & 1418-1957 & $1,2,3,4,8$ & $1-480$ & $481-728$ & 729-908 & 909-1090 & No & No & No & 1091-1951 \\
\hline 2 & OGG1-2C & NM_016827 & 1775 & $1-343$ & 344-931 & $932-1775$ & $1,2,3,8$ & $1-480$ & $481-728$ & 729-908 & No & No & No & No & 909-1769 \\
\hline 2 & OGG1-2d & NM_016828 & 2258 & $1-343$ & $344-1414$ & $1415-2258$ & $1,2,3,4,5,6,7,8$ & $1-480$ & $481-728$ & 729-908 & 909-1090 & $1091-1241$ & $1242-1291$ & 1292-1391 & $1392-2252$ \\
\hline 2 & OGG1-2e & NM_016829 & 2211 & $1-343$ & $344-1312$ & $1313-2211$ & $1,2,3,4,5,6,7,8$ & $1-480$ & $481-728$ & 729-908 & 909-1090 & $1091-1241$ & $1242-1291$ & $1292-1344$ & $1345-2205$ \\
\hline
\end{tabular}

Bold columns show differences among isoforms 
Table 2 Alternative splicing isoforms of OGG1 CDS to NCB

\begin{tabular}{|c|c|c|c|c|c|c|c|c|c|c|c|c|c|}
\hline \multirow[t]{2}{*}{ Type } & \multirow[t]{2}{*}{ Name } & \multicolumn{9}{|l|}{ mRNA } & \multicolumn{3}{|l|}{ CDS } \\
\hline & & $\begin{array}{l}\text { CDS in } \\
\text { mRNA }\end{array}$ & $\begin{array}{l}\text { From } \\
\text { exon } 1\end{array}$ & $\begin{array}{l}\text { From } \\
\text { exon } 2\end{array}$ & $\begin{array}{l}\text { From } \\
\text { exon } 3\end{array}$ & $\begin{array}{l}\text { From } \\
\text { exon } 4\end{array}$ & $\begin{array}{l}\text { From } \\
\text { exon } 5\end{array}$ & $\begin{array}{l}\text { From } \\
\text { exon } 6\end{array}$ & $\begin{array}{l}\text { From } \\
\text { exon } 7\end{array}$ & $\begin{array}{l}\text { From } \\
\text { exon } 8\end{array}$ & $\begin{array}{l}\text { nt length } \\
\text { in CDS }\end{array}$ & $\begin{array}{l}\text { identical to } \\
\text { OGG1-1a CDS }\end{array}$ & $\begin{array}{l}\text { Identical to } \\
\text { OGG1-2a CDS }\end{array}$ \\
\hline 1 & OGG1-1a & 344-1381 & $344-480$ & $481-728$ & $729-908$ & $909-1090$ & $1091-1241$ & $1242-1291$ & $1292-1381$ & No & 1038 & - & $1-949$ \\
\hline 1 & OGG1-1b & 344-1318 & $344-480$ & $481-728$ & 729-908 & 909-1090 & $1091-1241$ & $1242-1318$ & No & No & 975 & $1-951$ & $1-949$ \\
\hline 1 & OGG1-1C & $344-1576$ & $344-480$ & $481-728$ & 729-908 & $909-1090$ & $1091-1241$ & $1242-1291$ & $1292-1576$ & No & 1233 & $\begin{array}{l}\text { 1-948, 966-1055-1c: } \\
949-1038-1 a\end{array}$ & $1-948$ \\
\hline 2 & OGG1-2a & $344-1618$ & $344-480$ & $481-728$ & 729-908 & $909-1090$ & $1091-1241$ & $1242-1291$ & No & $1292-1618$ & 1275 & $1-949$ & - \\
\hline 2 & OGG1-2b & $344-1417$ & $344-480$ & $481-728$ & $729-908$ & $909-1090$ & No & No & No & $1091-1417$ & 1074 & $1-748$ & $\begin{array}{l}1-748,749-1074-2 c: \\
950-1275-2 a\end{array}$ \\
\hline 2 & OGG1-2C & $344-931$ & $344-480$ & $481-728$ & 729-908 & No & No & No & No & $909-931$ & 588 & $1-569$ & $1-569$ \\
\hline 2 & OGG1-2d & $344-1414$ & $344-480$ & $481-728$ & 729-908 & $909-1090$ & $1091-1241$ & $1242-1291$ & $1292-1391$ & $1392-1414$ & 1071 & $1-948$ & $1-948$ \\
\hline 2 & OGG1-2e & $344-1312$ & $344-480$ & $481-728$ & 729-908 & $909-1090$ & $1091-1241$ & $1242-1291$ & $1292-1312$ & No & 969 & $1-949$ & $1-949$ \\
\hline
\end{tabular}

Bold columns show differences among isoforms 
Table 3 Protein products of OGG1 isoforms according to NCBI

\begin{tabular}{|c|c|c|c|c|c|c|c|c|c|c|c|c|c|c|c|c|}
\hline Type & Name & $\begin{array}{l}\text { Protein } \\
\text { accession }\end{array}$ & $\begin{array}{l}\text { Amino } \\
\text { acids }\end{array}$ & $\begin{array}{l}\text { From } \\
\text { exon } 1\end{array}$ & $\begin{array}{l}\text { From } \\
\text { exon } 2\end{array}$ & $\begin{array}{l}\text { From } \\
\text { exon } 3\end{array}$ & $\begin{array}{l}\text { From } \\
\text { exon } 4\end{array}$ & $\begin{array}{l}\text { From } \\
\text { exon } 5\end{array}$ & $\begin{array}{l}\text { From } \\
\text { exon } 6\end{array}$ & $\begin{array}{l}\text { From } \\
\text { exon } 7\end{array}$ & $\begin{array}{l}\text { From } \\
\text { exon } 8\end{array}$ & $\begin{array}{l}\text { Identical to } \\
\text { OGG1-1a }\end{array}$ & $\begin{array}{l}\text { Identical to } \\
\text { OGG1-2a }\end{array}$ & MTS & NLS & OGG1 activity \\
\hline 1 & OGG1-1a & NP_002533 & 345 & $46^{\mathrm{a}}$ & $83^{a}$ & $60^{a}$ & 60 & $51^{a}$ & 16 & 29 & No & - & $1-316$ & 9 to 26 & $335-341$ & Active [6] \\
\hline 1 & OGG1-1b & NP_058212 & 324 & $46^{\mathrm{a}}$ & $83^{\mathrm{a}}$ & $60^{\mathrm{a}}$ & 60 & $51^{\mathrm{a}}$ & 24 & No & No & $1-317$ & $1-316$ & 9 to 26 & No & Active [11] \\
\hline 1 & OGG1-1c & NP_058213 & 410 & $46^{\mathrm{a}}$ & $83^{\mathrm{a}}$ & $60^{a}$ & 60 & $51^{\mathrm{a}}$ & 16 & 94 & No & $1-316$ & $1-316$ & 9 to 26 & No & ND \\
\hline 2 & OGG1-2a & NP_058214 & 424 & $46^{\mathrm{a}}$ & $83^{\mathrm{a}}$ & $60^{\mathrm{a}}$ & 60 & $51^{\mathrm{a}}$ & 16 & No & $108^{\mathrm{a}}$ & $1-316$ & - & 9 to 26 & No & Unresolved $[9,10]$ \\
\hline 2 & OGG1-2b & NP_058434 & 357 & $46^{\mathrm{a}}$ & $83^{\mathrm{a}}$ & $60^{a}$ & 60 & No & No & No & $108^{\mathrm{a}}$ & $1-249$ & $\begin{array}{l}1-249,250-357-2 b: \\
317-424-2 a\end{array}$ & 9 to 26 & No & ND \\
\hline 2 & OGG1-2C & NP_058436 & 195 & $46^{\mathrm{a}}$ & $83^{\mathrm{a}}$ & $60^{\mathrm{a}}$ & No & No & No & No & $6^{\mathrm{b}}$ & $1-190$ & $1-190$ & 9 to 26 & No & ND \\
\hline 2 & OGG1-2d & NP_058437 & 356 & $46^{\mathrm{a}}$ & $83^{\mathrm{a}}$ & $60^{\mathrm{a}}$ & 60 & $51^{a}$ & 16 & $34^{\mathrm{a}}$ & $6^{\mathrm{b}}$ & $1-316$ & $1-316$ & 9 to 26 & No & ND \\
\hline 2 & OGG1-2e & NP_058438 & 322 & $46^{\mathrm{a}}$ & $83^{\mathrm{a}}$ & $60^{\mathrm{a}}$ & 60 & $51^{\mathrm{a}}$ & 16 & 6 & No & $1-316$ & $1-316$ & 9 to 26 & No & ND \\
\hline
\end{tabular}

Bold columns show differences among isoforms

Abbreviations: MTS mitochondrial localization signal, RRMGHRTLASTPALWASI, NLS nuclear localization signal, KRRKGSK., ND not determined

${ }^{\mathrm{a}}$ indicates amino acids encoded cross a splice junction. ${ }^{\mathrm{b}}$ the same amino acid sequence. ${ }^{\mathrm{c}}$ the same amino aid sequence 
splice junction from exon 3, plus six amino acids and the stop codon) from exon 8 of the OGG1-2c mRNA is the last part of the CDS, resulting in a different amino acid sequence from that of OGG1-2a and OGG1-2b. The nt sequence $932-1775$ (844 bp) is the $3^{\prime}$-UTR and has a different length to the 3 '-UTRs from the OGG1-2a (540 bp) and OGG1-2b mRNAs (540 bp).

The OGG1-2d mRNA has exons 7-8. The whole nt sequence of exon 7, 1292-1391 (100 bp), in the OGG1-2d mRNA is a CDS. The nucleotide sequence of 1392-1414 (23 bp: the first two nucleotides cross a splice junction from exon 7, plus six amino acids and the stop codon) from exon 8 of the OGG1-2d mRNA is the last part of the CDS, and gives rise to the same six amino acid sequence as that of the OGG1-2c protein. The nt sequence of 1415-2258 (844 bp) is the 3'-UTR.

The OGG1-2e mRNA has exons 7 and 8 . The nucleotide sequence 1292-1312 (21 bp for six amino acids and the stop codon) from the first part of exon 7 of the OGG1-2e mRNA is the last part of the OGG1-2e CDS, resulting in an amino acid sequence that differs from the exon 7 CDS of OGG1-1a, OGG1-1c, and OGG1-2d. The nt sequence 1313-1344 is a part of the 3'-UTR. Exon 8 (861 bp) of the OGG1-2e mRNA is a continuous 3'-UTR. The whole exon 7 nt sequence 1292-1344 (53 bp) of the OGG1-2e mRNA is identical to part of the nt sequence 1339-1391 (53 bp) in exon 7 of the OGG1-2d mRNA.

\section{Conclusions}

Eight alternatively spliced isoforms of human 8-oxoguanine DNA glycosylase (OGG1) are registered with the NCBI. OGG1-1a is present in the nucleus, whereas the other seven isoforms are present in mitochondria. Recombinant OGG1-1a has been purified and its enzyme kinetics studied. The mitochondrial major OGG1 isoform, OGG1-2a (also named $\beta$-OGG1), has been purified; however, the OGG1 activity of this enzyme was unusual and has not been determined. Recently, we purified recombinant mitochondrial OGG1-1b and showed that it is an active OGG1 enzyme. We reported its enzyme kinetics and compared the results with the corresponding kinetics of OGG11a. The OGG1 activity of OGG1-1b was similar to that of OGG1-1a, except for the $k_{\mathrm{gl}}$ against 8-oxoG:A. The OGG1-1b mRNA was detected by RT-PCR in normal human lung tissue and lung cells lines. These results suggest that OGG1-1b is associated with 8-oxoG cleavage at least in human lung mitochondria, and the repair mechanism is similar to that of nuclear OGG1-1a. Currently, the other five mitochondrial OGG1 isoforms have not been purified.

\footnotetext{
Abbreviations

CDS: coding DNA sequence; 5'-UTR: Five prime untranslated region; OGG1: Human 8-oxoguanine DNA glycosylase; nt: Nucleotide; NCBI: the National Center for Biotechnology Information; $k_{\mathrm{g}}$ : the reaction rate constant
}

of the 8-oxoG glycosylase activity; $\mathrm{kgl}_{\mathrm{gl}}$ : the reaction rate constant of $\mathrm{N}$-glycosylase/DNA lyase activity; 3'-UTR: Three prime untranslated region.

\section{Competing interests}

The author declares that he has no competing interests.

Received: 21 April 2015 Accepted: 12 August 2015

Published online: 01 October 2015

\section{References}

1. Kasai H, Tanooka H, Nishimura S. Formation of 8-hydroxyguanine residues in DNA by X-irradiation. Gan. 1984;75:1037-9.

2. Panduri V, Liu G, Surapureddi S, Kondapalli J, Soberanes S, de Souza-Pinto NC, et al. Role of mitochondrial hOGG1 and aconitase in oxidant-induced lung epithelial cell apoptosis. Free Radic Biol Med. 2009:47:750-9.

3. Aburatani H, Hippo Y, Ishida T, Takashima R, Matsuba C, Kodama T, et al. Cloning and characterization of mammalian 8-hydroxyguanine-specific DNA glycosylase/apurinic, apyrimidinic lyase, a functional mutM homologue. Cancer Res. 1997;57:2151-6.

4. Nishioka K, Ohtsubo T, Oda H, Fujiwara T, Kang D, Sugimachi K, et al. Expression and differential intracellular localization of two major forms of human 8-oxoguanine DNA glycosylase encoded by alternatively spliced OGG1 mRNAs. Mol Biol Cell. 1999;10:1637-52.

5. Takao M, Aburatani H, Kobayashi K, Yasui A. Mitochondrial targeting of human DNA glycosylases for repair of oxidative DNA damage. Nucleic Acids Res. 1998;26:2917-22.

6. McKibbin PL, Kobori A, Taniguchi Y, Kool ET, David SS. Surprising repair activities of nonpolar analogs of 8-oxoG expose features of recognition and catalysis by base excision repair glycosylases. J Am Chem Soc. 2012;134:1653-61.

7. Lukina MV, Popov AV, Koval W, Vorobjev YN, Fedorova OS, Zharkov DO. DNA damage processing by human 8-oxoguanine-DNA glycosylase mutants with the occluded active site. J Biol Chem. 2013;288:28936-47.

8. Kasymov RD, Grin IR, Endutkin AV, Smirnov SL, Ishchenko AA, Saparbaev MK, et al. Excision of 8-oxoguanine from methylated $\mathrm{CpG}$ dinucleotides by human 8-oxoguanine DNA glycosylase. FEBS Lett. 2013;587:3129-34.

9. Roldán-Arjona T, Wei YF, Carter KC, Klungland A, Anselmino C, Wang RP, et al. Molecular cloning and functional expression of a human CDNA encoding the antimutator enzyme 8-hydroxyguanine-DNA glycosylase. Proc Natl Acad Sci USA. 1997;94:8016-20.

10. Hashiguchi K, Stuart JA, de Souza-Pinto NC, Bohr VA. The C-terminal alphaO helix of human Ogg1 is essential for 8-oxoguanine DNA glycosylase activity: the mitochondrial beta Ogg1 lacks this domain and does not have glycosylase activity. Nucleic Acids Res. 2004;32:5596-608.

11. Ogawa A, Watanabe T, Shoji S, Furihata C. Enzyme kinetics of an alternative splicing isoform of mitochondrial 8-oxoguanine DNA glycosylase, OGG1-1b, and compared with the nuclear OGG1-1a. J Biochem Mol Toxicol. 2015;29:49-56

12. Kohno T, Shinmura K, Tosaka M, Tani M, Kim SR, Sugimura H, et al. Genetic polymorphisms and alternative splicing of the OGG1 gene, that is involved in the repair of 8-hydroxyguanine in damaged DNA. Oncogene. 1998;16:3219-25.

13. Dorszewska J, Kempisty B, Jaroszewska-Kolecka J, Rózycka A, Florczak J, Lianeri $M$, et al. Expression and polymorphisms of gene 8-oxoguanine glycosylase 1 and the level of oxidative DNA damage in peripheral blood lymphocytes of patients with Alzheimer's disease. DNA Cell Biol. 2009;28:579-88.

14. Janssen K, Schlink K, Götte W, Hippler B, Kaina B, Oesch F. DNA repair activity of 8-oxoguanine DNA glycosylase 1 (OGG1) in human lymphocytes is not dependent on genetic polymorphism Ser326/Cys326. Mutat Res. 2001:486:207-16.

15. Bjørås M, Seeberg E, Luna L, Pearl LH, Barrett TE. Reciprocal "flipping" underlies substrate recognition and catalytic activation by the human 8-oxo-guanine DNA glycosylase. J Mol Biol. 2002;317:171-7.

16. Anderson PC, Daggett V. The R46Q, R131Q and R154H polymorphs of human DNA glycosylase/beta-lyase hOgg1 severely distort the active site and DNA recognition site but do not cause unfolding. J Am Chem Soc. 2009;131:9506-15.

17. Su YH, Lee $Y L$, Chen SF, Lee $Y P$, Hsieh YH, Tsai JH, et al. Essential role of $\beta$-human 8-oxoguanine DNA glycosylase 1 in mitochondrial oxidative DNA repair. Environ Mol Mutagenesis. 2013;54:54-64. 